Alan J. Stevens

Brookhaven National Laboratory

Upton, NY 11973 


\section{Estimated Shielding Requirements for the PHENIX Detector}

\section{Introduction}

$\therefore$

This note describes a series of calculations whose purpose was to estimate the radiation dose equivalent levels in the assembly area and counting house regions in the vicinity of the 8 o'clock hall. The shield thickness required is determined by the possibility of a design basis fault in or near the hall. A design basis fault is loss of the full beam on any magnet which is at or near the limiting aperture of the machine and loss of one-half of the full beam on any other magnet. At the time of this writing, the objects which are "at or near the limiting aperture" are considered to be the high beta quadrupoles, the extraction and injection magnets (septa and kickers), and the internal dump. The RHIC requirement is that passive shielding should be sufficient to limit the dose equivalent from such a fault to $\leq 500 \mathrm{mrem}$ to a radiation worker. The regions under discussion here will be posted as radiation areas.

Calculations were performed with the hadron cascade monte carlo program CASIM., 1,2 The quantity actually calculated by CASIM is the star density per interacting primary particle (SD). This quantity, which is the number of interactions per unit volume per primary of all hadrons above the CASIM threshold of $0.3 \mathrm{GeV} / \mathrm{c}$, can be related to total dose equivalent if one assumes an equilibrium spectrum. For the purposes of this note, we will assume that the relationship is given by the following: Dose (rem/primary) $=4.5 \times 10^{-7} \times \mathrm{L} \times \mathrm{SD}$ where $\mathrm{L}$ is the (high energy) neutron interaction length in $\mathrm{cm}$. and SD is in units of $s t a r s / \mathrm{cm}^{3}$ primary. This is twice the normal CASIM star density to rem conversion constant which follows the recommendation of the "RADCON Manual"3 to assume an increased quality factor for low energy neutrons for design purposes.

\section{Geometry of the Calculation}

The geometry of the calculation in the 8 o'clock hall region is sketched in Fig. 1. This sketch is adapted from a more detailed one provided by the PHENIX collaboration. The elements of the PHENIX detector that are approximated are the beam pipe (not shown in the figure), the magnet pole/coil steel, the nose cone/piston/donut assembly, and the muon detector. The material is taken to be azimuthally symmetric around the beam axis although only one side is shown in Fig. 1. The shield wall separating the detector from the assembly area is taken to be light concrete. The material shielding the counting house was initially assumed to be soil, but this assumption will be modified in section $\mathrm{V}$ below. The material in the muon detector was treated as half-density steel, with the other components shown approximated as normal density steel. Near the end of the right hand side of Fig. I the accelerator enclosure decreases from the $5.3 \mathrm{~m}$ radius shown to about $3.05 \mathrm{~m}$ radius.

The approximation of the magnets is shown in Fig. 2. As in previous calculationst, the approximation is made that only one ring of magnets exists whose axis coincides with the tunnel axis. The beam pipe is exagrgerated in thickness (but reduced in density) to minimize "stepping 
over" the pipe during transport. The magnetic fields are taken into account within the apertures of the magnets but ignored in the coil/yoke regions. The origin of the coordinate system in Fig. 2 , $Z=0$, is the beginning of $Q 2$. In this system the hall begins at $23 \mathrm{~m}$ which is the $Z=0$ point in Fig. 1

\section{Description of the Calculations}

In all hadron shielding calculations done to date, any differences between $250 \mathrm{GeV} / \mathrm{c}$ protons and $100 \mathrm{GeV} / \mathrm{u} \mathrm{Au}$ ions - normalized to the number of particles in the beam - has been so small as to be essentially unobservable. ${ }^{5}$ Since the heavy ion version of CASIM is much slower than the proton version, only $250 \mathrm{GeV} / \mathrm{c}$ protons were considered.

It should be clear from Fig. 1 that a variety of faults must be considered. The assembly area is "exposed" to faults on the magnets on both sides of this figure; both the clockwise and counter-clockwise beam directions must be considered. The counting house is exposed to faults on the magnets on the left hand side of Fig. 1 (only DX is indicated in the figure) from the clockwise beam and to faults on the right hand side magnets from both beams. The fact that the tunnel radius decreases to $3.05 \mathrm{~m}$ at about $\pm 25 \mathrm{~m}$ from the crossing point offers some protection from upstream magnet faults.

A full energy fault on a magnet is simulated by forcing an interaction to occur in the midplane beam pipe uniformly along the length of a magnet. This approximation is justified in Ref. [4]. In addition to magnet faults, a simulation of the beam scraping the beam pipe in the hall was also considered. In this case, the beam was forced to interact along a $3 \mathrm{~m}$ length of the pipe in the nose cone-piston region.

As mentioned above, the geometry assumes cylindrical symmetry. The material distribution described in the preceding section corresponds (more or less) to the detector on the horizontal mid-plane. The quantity calculated is the azimuthally averaged star density. To correct this for magnetic field effects, an accounting is made in the shield material of left-right versus up-down stars. If $f$ is the fraction of left-right stars to total stars, the azimuthally averaged star density is multiplied by $2 f$ before converting to the dose equivalent estimate. This correction is never higher than 1.3 .

\section{Results}

\section{(A) Assembly Area}

Fig. 3 shows the azimuthally averaged star density vs. the $Z$ coordinate given in Fig. 1 at the back of the assembly area wall (1.6m depth of light concrete) from three sources: (1) The DX magnet on the LHS (left hand side) of Fig. 1 with beam in clockwise direction [circles]; (2) DX on the RHS of Fig. 1 with counter-clockwise beam [crosses]; and (3) beam pipe at the nose cone-piston location with counter-clockwise beam [triangles]. The three sources are illustrated in Fig. 4. As in the case of the calculations done for STAR ${ }^{4}$, the corresponding star density for 
sources further upstream fall off rapidly; for the DO magnet on the LHS, for example, the maximum star density is lower than the peak shown in Fig. 4 by slightly over a factor of 2 .

The source of the RHS DX shows the effect of the muon wall shielding. The peak star density here clearly corresponds to radiation which can miss this wall as illustrated in Fig. 4. At smaller values of $Z$ in Fig. 3, comparison of the two DX sources in Fig. 3 shows that the muon wall reduces the dose by about a factor of 5 .

The worst case is clearly the DX on the left hand side of Fig. 4. The maximum dose equivalent at design intensity given by:

$$
\begin{aligned}
\text { Maxmrem } / \text { fault } & =2.6 \times 10^{-9} \text { star } / c c / p \times 1.8 \times 10^{-2} \text { mrem } / \text { star } / c c \times 1.3 \times 2.85 \times 10^{12} p \\
& \cong 173 \mathrm{mrem}
\end{aligned}
$$

In this expression, the $1.8 \times 10^{-2} \mathrm{mrem} / \mathrm{star} / \mathrm{cc}$ is twice the standard star density to dose equivalent conversion factor in light concrete as discussed above, 1.3 is the magnetic field enhancement factor, and $2.85 \times 10^{12}$ is half the design intensity which is the design basis fault assumption for DX. Although the $1.6 \mathrm{~m}$ thick light concrete wall beginning at $7.8 \mathrm{~m}$ from the beam line is sufficient for the RHIC design intensity, it does not suffice at 4 times the design intensity. A $6 \mathrm{ft}$. thick wall $(\sim 183 \mathrm{~cm})$ reduces the maximum dose at the design intensity to $105 \mathrm{mrem}^{6}$ which is sufficient for 4 times the design value.

At the time of this writing, the possibility exists of a second muon arm being present in the PHENIX detector. As noted above, the muon arm provides considerable shielding for faults on the RHS of Fig. 1. If a second muon arm is present, the worst case (see Fig. 3) becomes scraping on the beam pipe within the hall. The difference between the LHS DX and the beam pipe is a factor of two when the absence of a magnetic field enhancement factor in the beam pipe loss is taken into account. This is equivalent to $1 \mathrm{ft}$ of concrete. If a second muon arm is present the requirement is therefore reduced to five feet of concrete.

\section{(B) Counting House}

In this section, the Counting House region will be considered to be separated from the accelerator by a solid wall of soil as shown in Fig. 1. In the next section, this picture will be modified, and a correction made to the results obtained here.

Fig. 5 illustrates the calculations that were done for sources illuminating the back edge of the counting house shield. The clockwise beam is a source of faults on both DX's. As shown in Fig. 5, a fault on the LHS DX can "punch through" the end wall separating the assembly area from the counting house region. Faults of the counter-clockwise beam were considered on the Right Hand Side magnets D0, Q1, and Q2.

The punch through from the LHS DX has a maximum azimuthally averaged star density of $7,10^{-11} \mathrm{stars} / \mathrm{cc} / \mathrm{p}$ in soil at the back wall of the shield shown. This maximum value occurs at 
the $Z=21 \mathrm{~m}$ position in Fig. 1 and drops to half this value by $Z=22.5 \mathrm{~m}$ The maximum value is the equivalent of $9.3 \times 10^{-10}$ in concrete which is a factor of 2.8 lower than was calculated in the last section, or about $62 \mathrm{mrem}$ at the design intensity. Not un che avou

As should be clear from Fig. 5, the counting house has a thick shield for faults from the counter-clockwise beam. The worst case at the back of the shield is obtained from a fault on Q2. The maximum star density in this case in soil is about $5 \times 10^{-11} \mathrm{stars} / \mathrm{cc} / \mathrm{p}$. The rem per fault at design intensity is the following:

$$
\begin{aligned}
\text { Max mrem / fault } & =5 \times 10^{-11} \text { star } / c c / p \times 2.4 \times 10^{-2} \text { mrem } / \text { star } / c c \times 1.15 \times 5.7 \times 10^{12} p \\
& \cong 8 \mathrm{mrem}
\end{aligned}
$$

Here, $2.4 \times 10^{-2}$ is the star to dose conversion in sand, 1.15 is the magnetic field enhancement factor, and - because Q2 is a high $\beta$ quadrupole - the full beam intensity rather than half the beam intensity defines the design basis fault.

However, as discussed in the next section, the counting house shield is not the continuous block of soil assumed above, since a labyrinth must be constructed in this region. This introduces a "hole" in the shield which means that the 8 mrem calculated above is not a relevant number.

\section{Labyrinth}

The muon detector closes off access to or from the tunnel on the right hand side of Fig. 1. The tunnel therefore becomes a dead-end on this side and NFPA 101 regulations require an escape path within $50 \mathrm{ft}$. of the dead end. ${ }^{7}$ For this purpose an escape labyrinth must exist between the tunnel and the counting house.

The labyrinth is shown in Fig. 6 . A $3 \mathrm{ft}$. wide aisle is shown by the solid lines. The dashed lines indicate the presence of 1 foot of (light) concrete which is assumed to constitute the walls of the labyrinth. Two constraints have been adopted in making the sketch shown there. First, the entrance position shown is essentially "up against" the end wall where the tunnel narrows. A labyrinth whose entrance corresponds to the position of an existing doorway was examined and found to be inferior to the one sketched in Fig. 6 . The second constraint is that the exit has been kept well away from the punch though coming from the LHS DX. As mentioned in the last section, this punch through falls rapidly as a function of the beam coordinate. Introducing the hole shown in the shield wall does not affect the punch through.

The geometry shown in Fig. 6 was made part of a CASIM calculation. In this case, the sources examined (all on the right hand side of Fig.6) were the clockwise beam faulting on DX and D0 and the counter-clockwise beam faulting on D0 and Q2. The results of these calculations are given below. Such a calculation neglects two "low energy" components of the radiation field behind the wall which are illustrated in Fig. 7. The ray shown in this figure entering the labyrinth from the tunnel interior illustrates the classic low energy transmission component which is amenable to evaluation by employing labyrinth formula. The sccond ray, shown "punching 
through" the interior labyrinth wall and being transmitted down the third leg of the labyrinth, is supposed to represent the low energy component ( $<10 \mathrm{MeV}$ say) which enters the third leg from the point shown. Since CASIM does not transport low energy neutrons, the transmission along the duct represented by the third leg will be treated separately and added to the dose obtained by the CASIM calculation.

\section{(A) CASIM Results}

Fig. 8 shows the azimuthally averaged star density in soil for the two counter-clockwise magnets at the back of the counting house. The coordinate system here is the same as in Fig.'s 6 and 7. The peak values - at the position of the labyrinth exit — are both the same which implies that Q2 is the worst case since the full beam is allowed to fault here. Note that the Q2 points show a second peak of smaller magnitude which corresponds to the thin shield represented by the first leg of the labyrinth. For D0, this is in a slightly backward direction and does not show within the statistical errors. At the exit of the labyrinth the dose equivalent estimate at design intensity is:

$$
\begin{aligned}
\text { mrem / fault } & =6.5 \times 10^{-10} \text { star } / c c / p \times 2.4 \times 10^{-2} \text { mrem } / \text { star } / c c \times 1.15 \times 5.7 \times 10^{12} p \\
& =102 \mathrm{mrem} .
\end{aligned}
$$

Fig. 9 shows the results for the clockwise sources, DX and D0. The radiation from DX peaks at the exit of the labyrinth but is smaller (even per proton) than the counter-clockwise source. The peak from DO is higher and clearly corresponds to radiation penetrating the first labyrinth leg. The highest dose level here is:

$$
\begin{aligned}
\text { mrem } / \text { fault } & =5.6 \times 10^{-10} \text { star } / c c / p \times 2.4 \times 10^{-2} \text { mrem } / \text { star } / c c \times 1.30 \times 2.85 \times 10^{12} p \\
& =50 \mathrm{mrem}
\end{aligned}
$$

\section{(B) Low Energy Components}

As mentioned above, the dose at the labyrinth exit has two additional "low energy" components. These are estimated by employing the "universal curves" of Goebel ${ }^{8,9}$ for a point source off axis. ${ }^{10}$ Given a source term at the entrance of a labyrinth, the Goebel formula for attenuation $A$ are given by:

$$
\begin{array}{ll}
A=\frac{1}{1+2.5 \sqrt{d}+0.17 d^{1.7}+0.79 d^{3}} & \text { for the first leg and } \\
A=\frac{1}{1+2.8 d(1.57)^{d+2}} \quad \text { for subsequent legs. }
\end{array}
$$

where $d$ is the length of the labyrinth leg expressed in units of the square root of the labyrinth area 


\section{(B.1) Labyrinth "Proper" Component}

I have followed the procedure of Gollen ${ }^{11}$ who uses $85 \%$ of the dose equivalent deduced from the entrance CASIM star density as the source term for the multi-legged labyrinth calculations. The worst case turns out to be DX (clockwise) where the entrance star density gives a source term of $2.8 \times 10^{-9} \mathrm{mrem} / \mathrm{p}$. The leg lengths, assuming a $3 \mathrm{ft}$. by $7 \mathrm{ft}$. height are the following: $d_{1}=1.72, d_{2}=2.96$, and $d_{3}=1.24$. The formula above give an overall reduction factor of $1.1 \times 10^{4}$. The dose estimate at design intensity is therefore 0.73 mrem which is clearly negligible. The dose from Q2, which is the worst case from the CASIM dose equivalent, is about 0.60 mrem. ${ }^{12}$

\section{(B.2) Low Energy "Punch Through"}

This component is estimated by taking the low energy part of the CASIM dose in the concrete at the point where the punch-through exits the tunnel wall in Fig. 7, and attenuating this component by the first leg formula of Goebel. If one takes $10 \mathrm{MeV}$ as the dividing line, $58 \%$ of the dose is below this value. ${ }^{13}$

The worst case is again Q2. At the exit of the concrete wall the azimuthally averaged star density is $3.1 \times 10^{-9} \mathrm{star} / \mathrm{cc} / \mathrm{p}$. The dose equivalent at the labyrinth exit is then:

$$
\begin{aligned}
\text { mrem } / \text { fault } & =3.1 \times 10^{-9} \times 1.15 \times .58 \times \frac{1}{10.15} \times 1.8 \times 10^{-2} \times 5.7 \times 10^{12} \\
& =21 \mathrm{mrem}
\end{aligned}
$$

In this expression, 1.15 is the usual magnetic field enhancement factor and the reduction of 10.15 follows from the first leg attenuation equation of Goebel given above with $d=1.90$.

\section{(C) Total Dose Equivalent}

At the exit of the labyrinth, the total dose nominally involves the sum of the above components. Although there is likely some "double counting" when adding the CASIM dose to the punch-through, this is the conservative procedure. At 4 times the design intensity, a fault on Q2 produces a worst-case:

$$
4 \times(102+21+0.6) \cong 494 \text { mrem } .
$$

Although this meets the 500 mrem criteria, it does so with no room to spare. For this reason, it is recommended that the labyrinth interior near the labyrinth be solid concrete as shown in Fig. 10. This provides (except for the very small multi-leg component) a safety factor of about $2^{14}$, and puts the calculated dose well within the design criteria. 


\section{Summary}

Calculations of dose equivalent were made to determine the shield wall thickness required for the PHENIX detector in the 8 o'clock hall. A minimum $6 \mathrm{ft}$. light concrete shield wall separating the 8 o'clock hall from the adjacent assembly area (5 ft. if a second muon arm is present) is required. The estimated dose equivalent in a design fault situation behind this shield wall is 420 mrem at 4 times the design intensity. No design for access between the hall and the assembly area has yet been evaluated.

Calculations were also made of the dose equivalent in a counting house region which will be constructed immediately adjacent to the assembly area. The dose here is dominated by an OSHA requirement to provide an emergency tunnel exit. A configuration is recommended which is calculated to have about $250 \mathrm{mrem}$ in the design basis fault scenario. A sketch of the recommended shielding configuration is shown in Fig. 10.

\section{$\underline{\text { References/Footnotes }}$}

1. A. Van Ginneken, "CASIM; Program to Simulate Hadron Cascades in Bulk Matter," Fermilab FN-272 (1975).

2. A.J. Stevens, "Improvements in CASIM; Comparison with Data," AGS/AD/Tech. Note,No. 296 (1988).

3. U.S. Department of Energy Radiological Control manual, DOE/EH-0256T (1992).

4. A. J. Stevens, "Local Shielding Requirements for the STAR Detector," RHIC/DET Note 5, (1992).

5. This also follows from simple formula for transverse shielding wherein the maximum dose scales as the 0.8 power of the beam energy. The relative dose, given the approximation that an Au ion is 197 pseudo-independent nucleons, is then expected to scale as $(250 / 100)^{0.8} \times(100 / 197)$ where the 100 simply reflects the fact that there are 100 more $250 \mathrm{GeV} / \mathrm{c}$ protons in the beam than $\mathrm{Au}$ ions. This scaling factor is essentially unity.

6. The dose in transverse geometries scales as $\exp (-d / \lambda) / \mathrm{R}_{\mathrm{t}}{ }^{2}$ where $\mathrm{R}_{\mathrm{t}}$ is the transverse distance to the back of the enclosure of thickness $d$ and $\lambda$ is an attenuation length. For light concrete, 50.2 $\mathrm{cm}$. is a reasonable value for $\lambda$. An alternative to making the wall thicker than originally shown would be form it from heavy concrete which has a $\lambda$ value of $40.8 \mathrm{~cm}$. For sand with density 1.8 $\mathrm{g} / \mathrm{cc}$ (assumed to form most of the material shielding the counting house) $\lambda=67 \mathrm{~cm}$.

7. J. Levesque, private communication. 
8. K. Goebel, G.R. Stevenson, J.T. Routti, and H.G. Vogt, "Evaluating Dose Rates Due to Neutron Leakage Through the Access Tunnels of the SPS," CERN Internal Report LABIIRA/Note/75-10 (1975).

9. G. R. Stevenson, "Neutron Attenuation in Labyrinths, Ducts and Penetrations at High-Energy Proton Accelerators," CERN Internal Report TIS-RP/182/CF (1987).

10. Although the sources appear to be on-axis in the sketches shown in this note, the labyrinth is, in fact, at a lower elevation that the magnets. The center of the labyrinth is 22.5 below the midplane of the magnets. This fact represents a slight conservatism in the estimates presented here. However, even if the entrance were aligned with the beam axis, the source is still quite diffuse. The radiation source at the labyrinth entrance is initiated by evaporation neutrons in the magnet steel. Although not presented in this note, calculation of the spatial distribution of such a source on the interior wall indicates substantial longitudinal spread which means the geometry is quite unlike a "point source" of radiation.

11. P. Gollon, "Shielding of Multi-Leg Penetrations into the RHIC Collider," May, 1994 (unpublished).

12. As previously, the assumption is always made that the design fault on Q2 is $5.7 \times 10^{12}$ protons and half this amount on other magnets. The entrance dose equivalent in mrem per proton is lower on Q2 by a factor of 2.5 from DX.

13. A Van Ginneken and M. Awschalom, "High Energy Particle Interactions in Large Targets," FNAL, Batavia (1975). This result follows from Fig. VI. 12 or VI.13 of this reference.

14. This follows from the fact that the wall between the tunnel interior and the second labyrinth leg in Fig. 6 or 7 has $4.4 \mathrm{ft}$. of sand. The substitution of concrete for sand reduces the dose by a factor of 0.86 per foot. See footnote [6] above: 


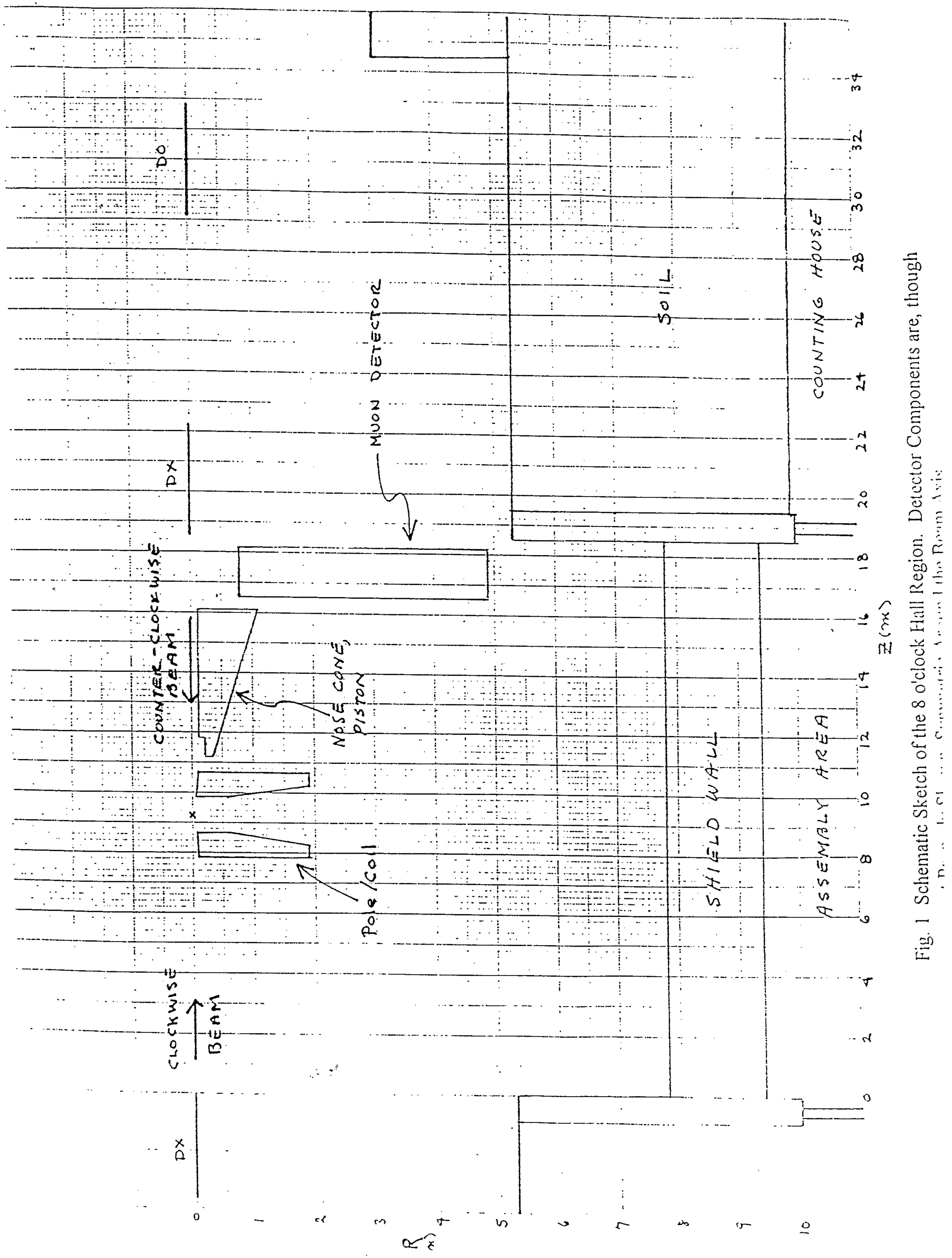



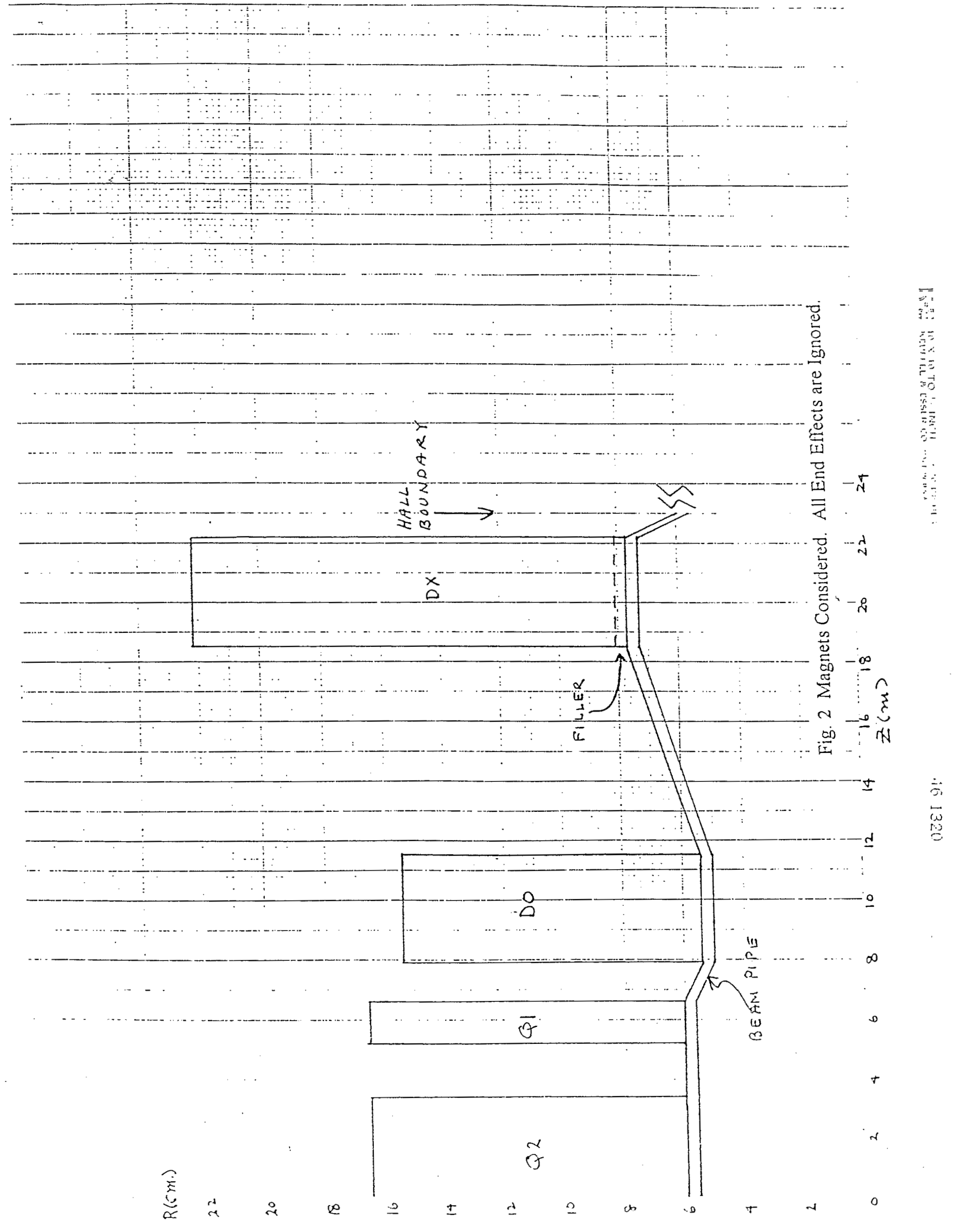


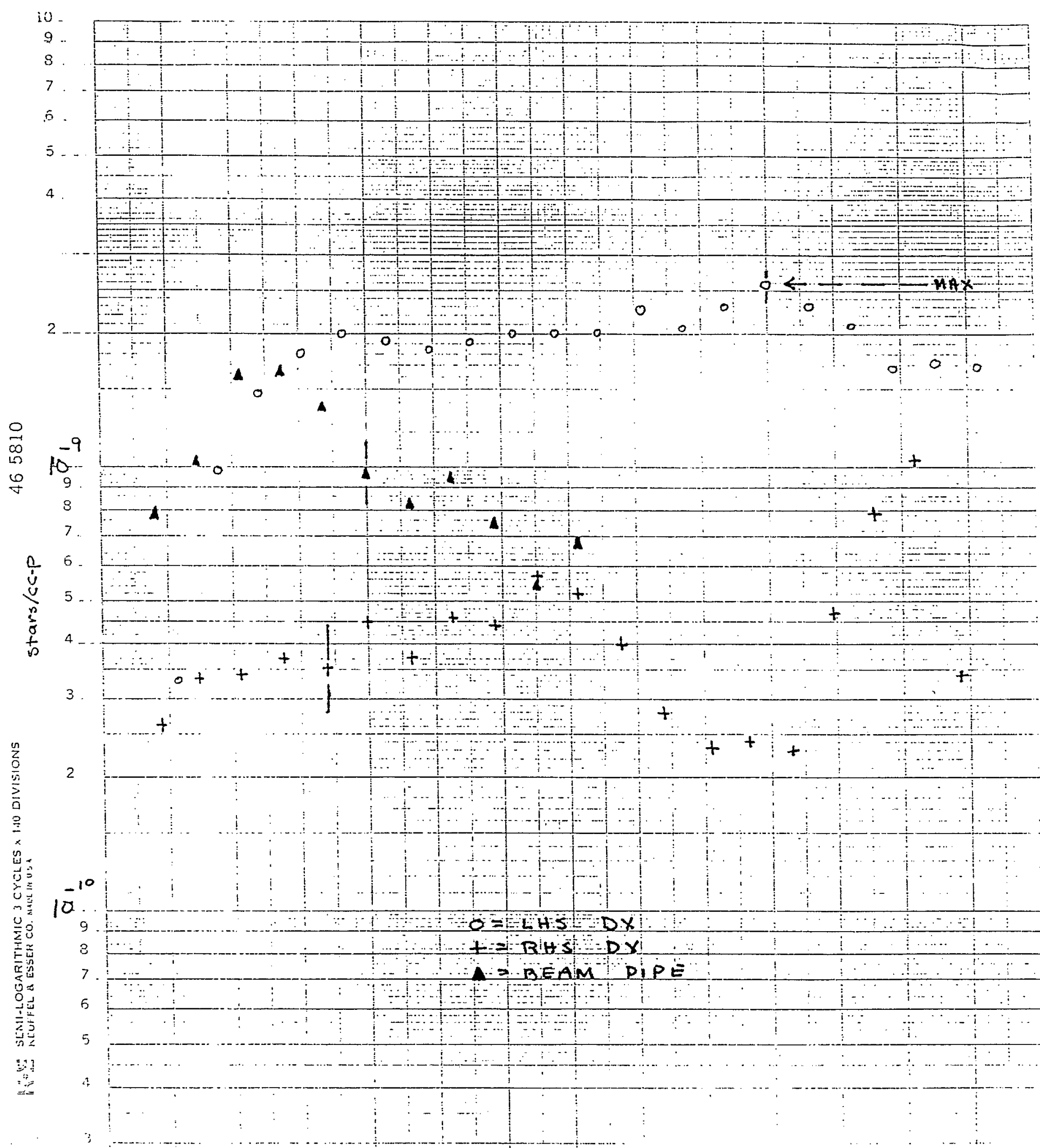

Fig. 3 Azimuthally Averaged Star Density vs. Position (Fig. 1) Behind Assembly Area Shield Wall (1.6m Depth) for the Three Sources Indicated. See Fig. 4. "Typical" Statistical Errors Shown. 


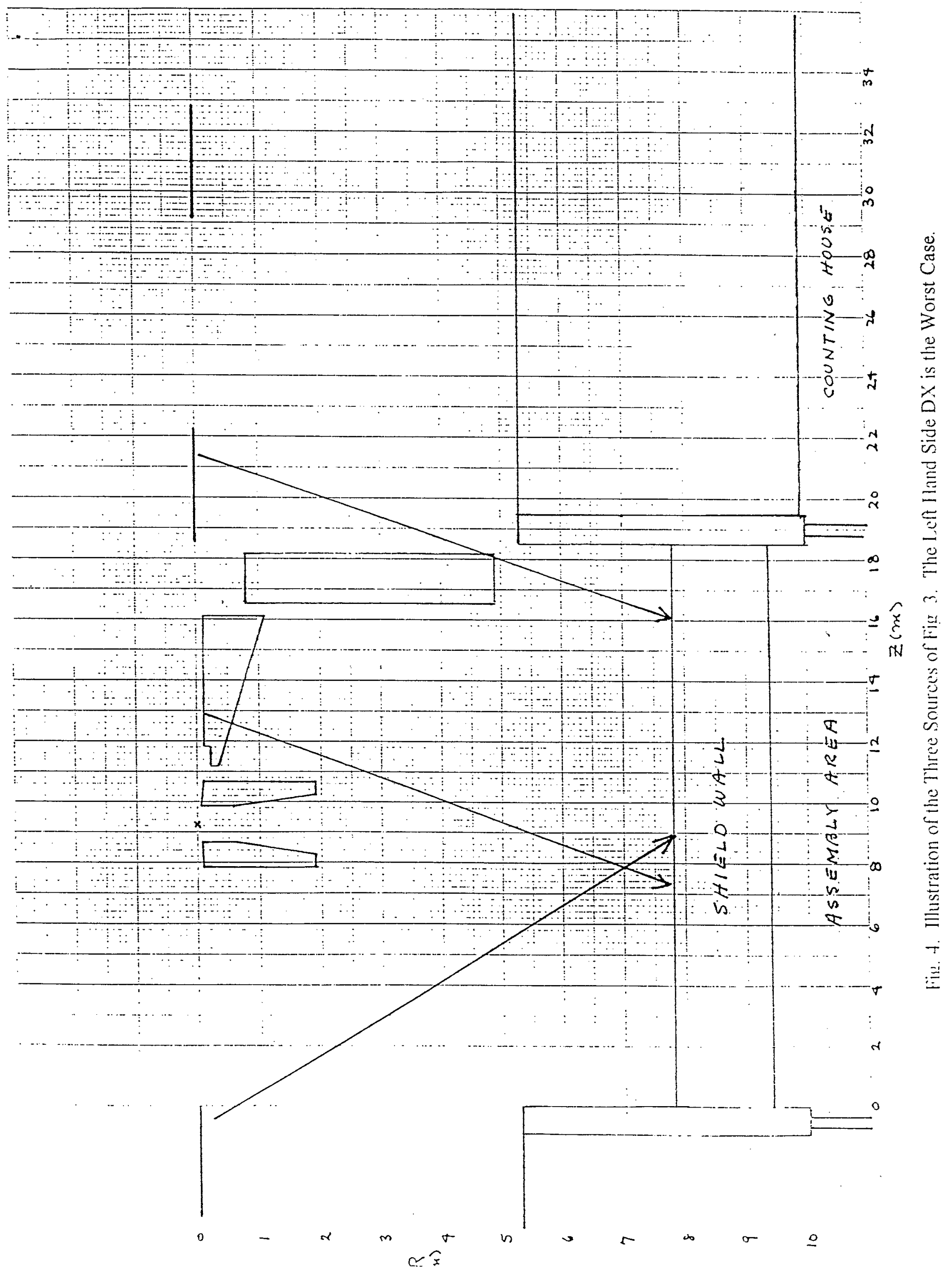




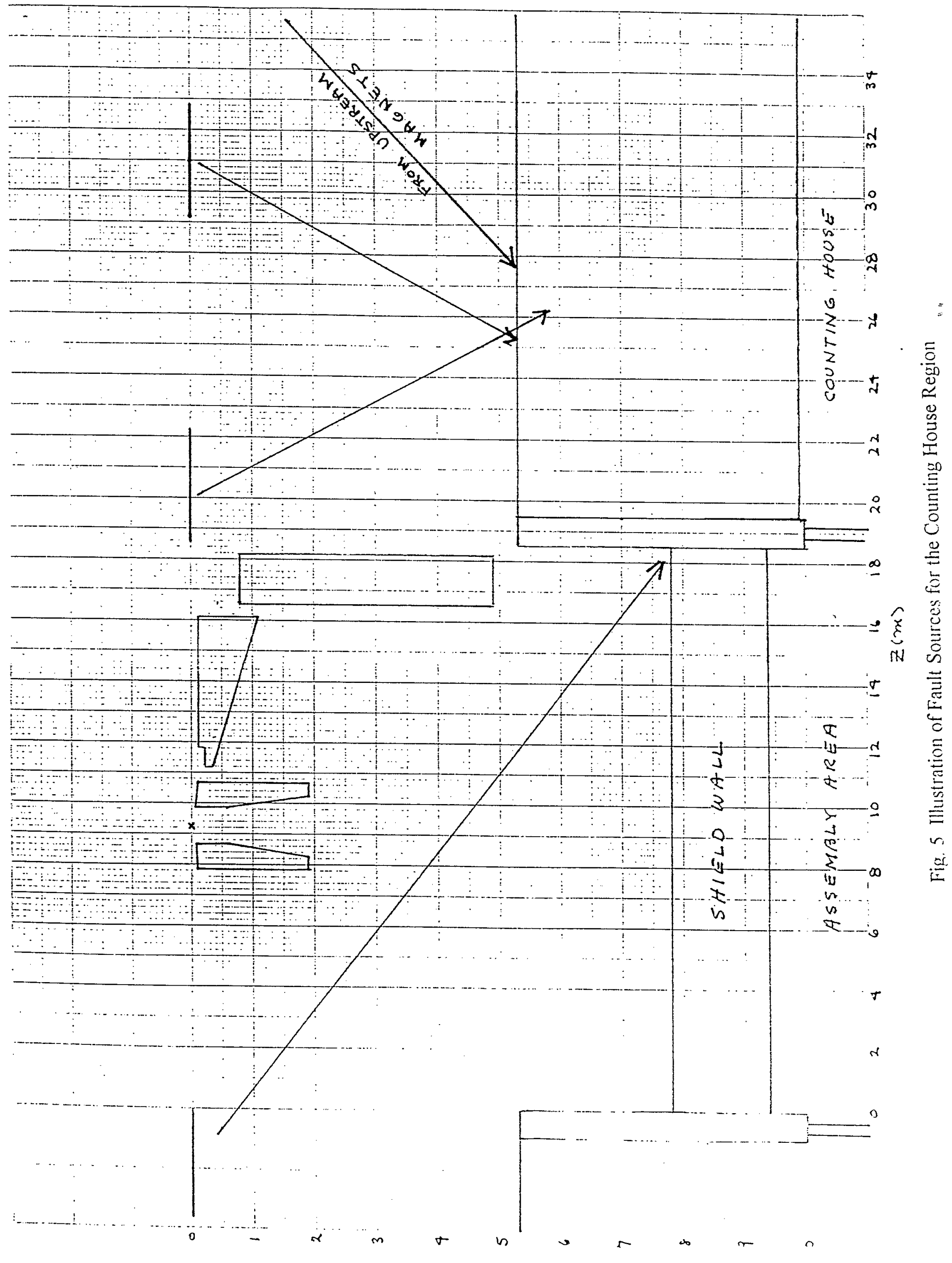




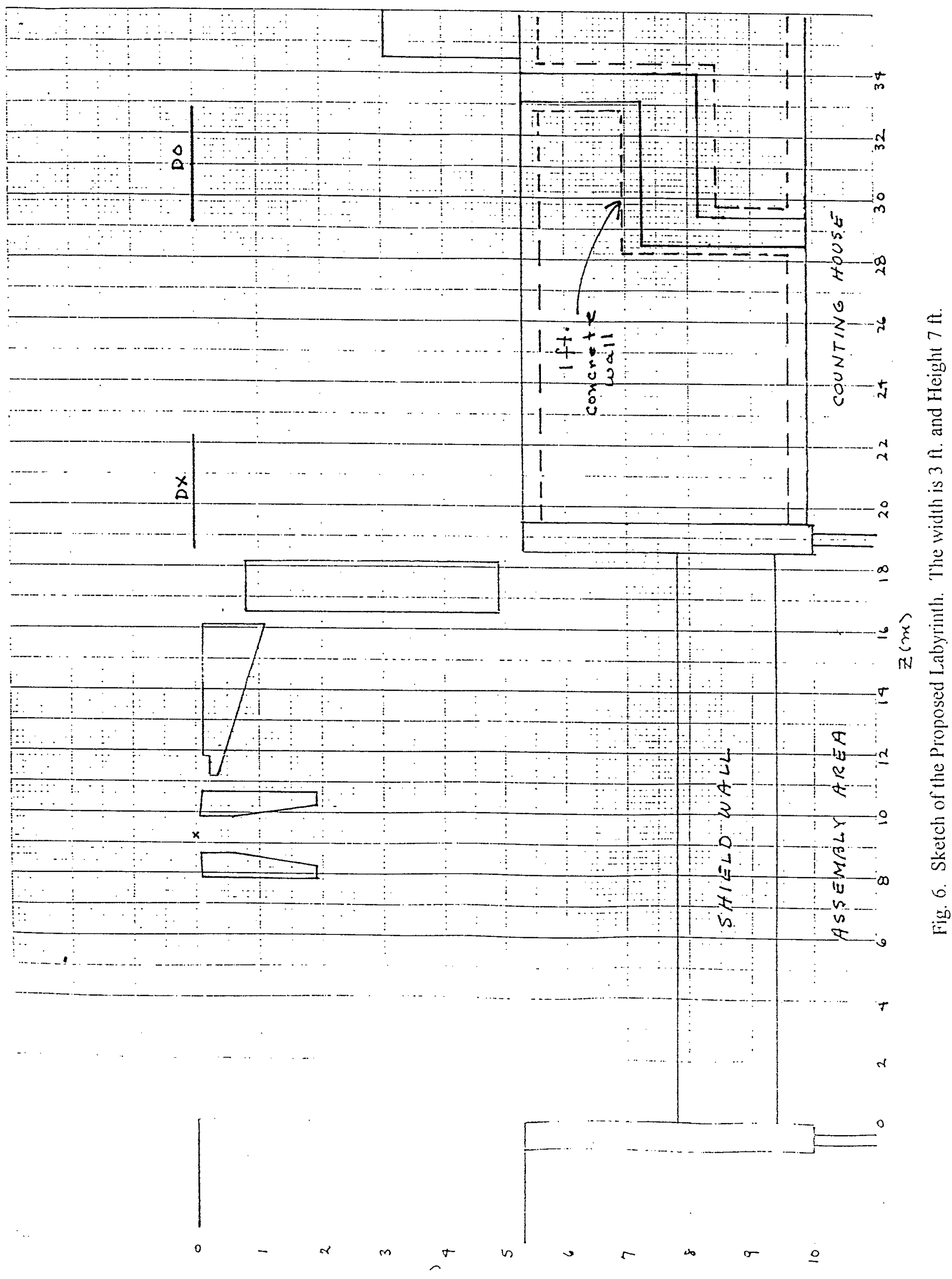




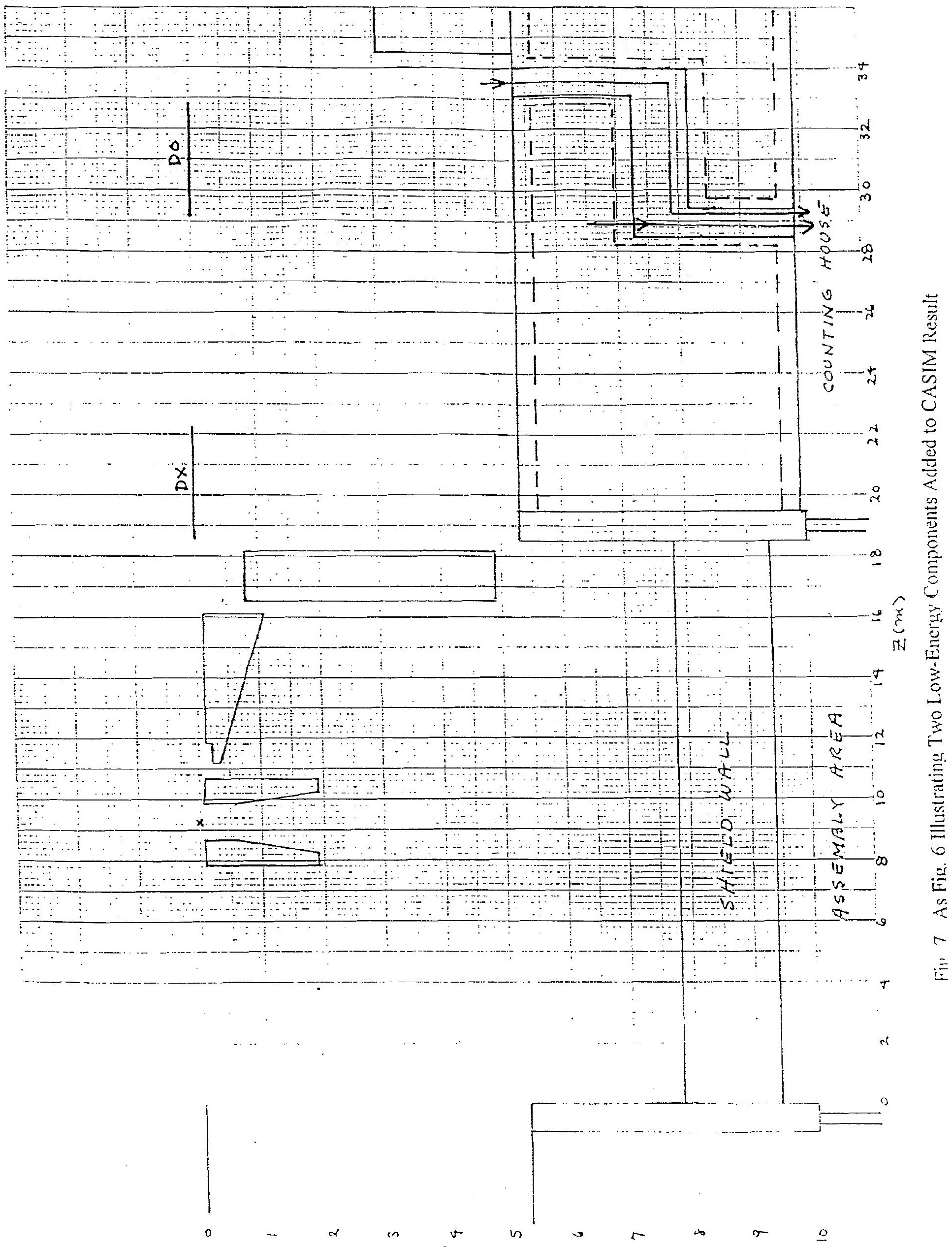




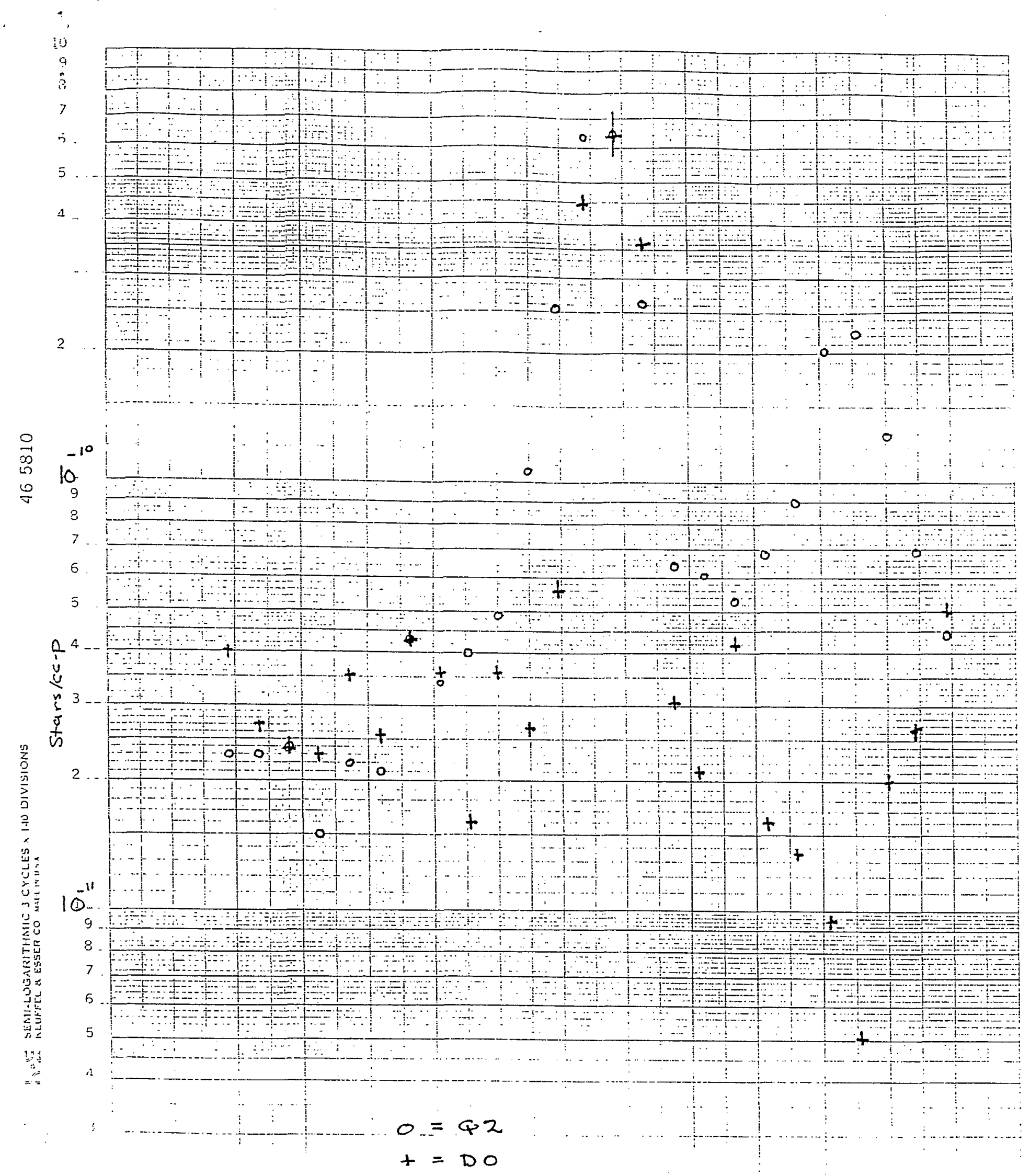

Fig. 8. Azimuthally Averaged Star Density vz. Z. at the Back of the Counting House $(R=9.9 \mathrm{~m})$ from the Sources Indicated.

The Beams are in the $-Z$ Direction Here. 


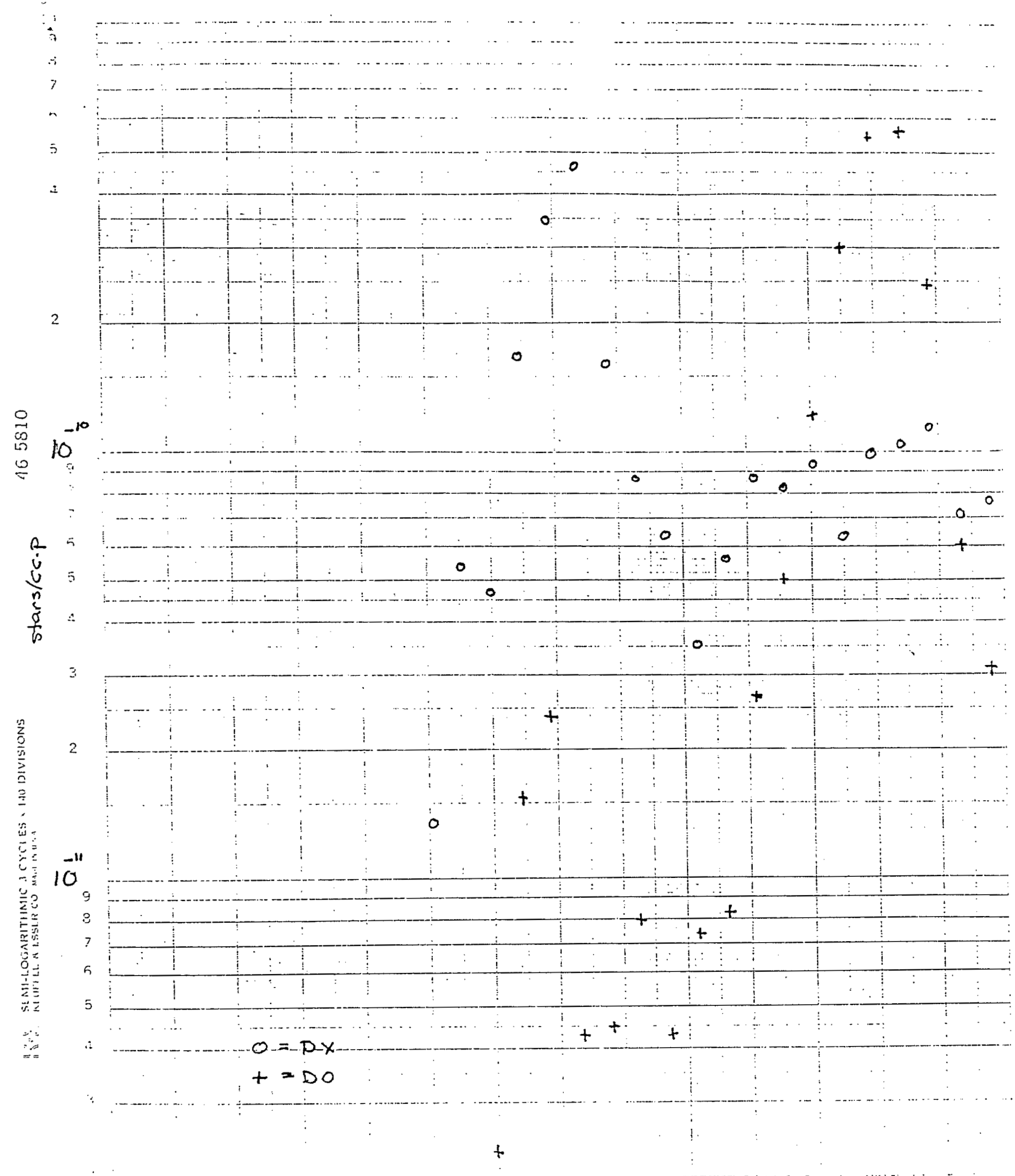

Fig. 9. Azimuthally Averaged Star Density vs. $Z$ at the Back of the Counting House $(R=9.9 \mathrm{~m})$ for the Sources Indicated. The Beam is in the $+Z$ Direction Here. 


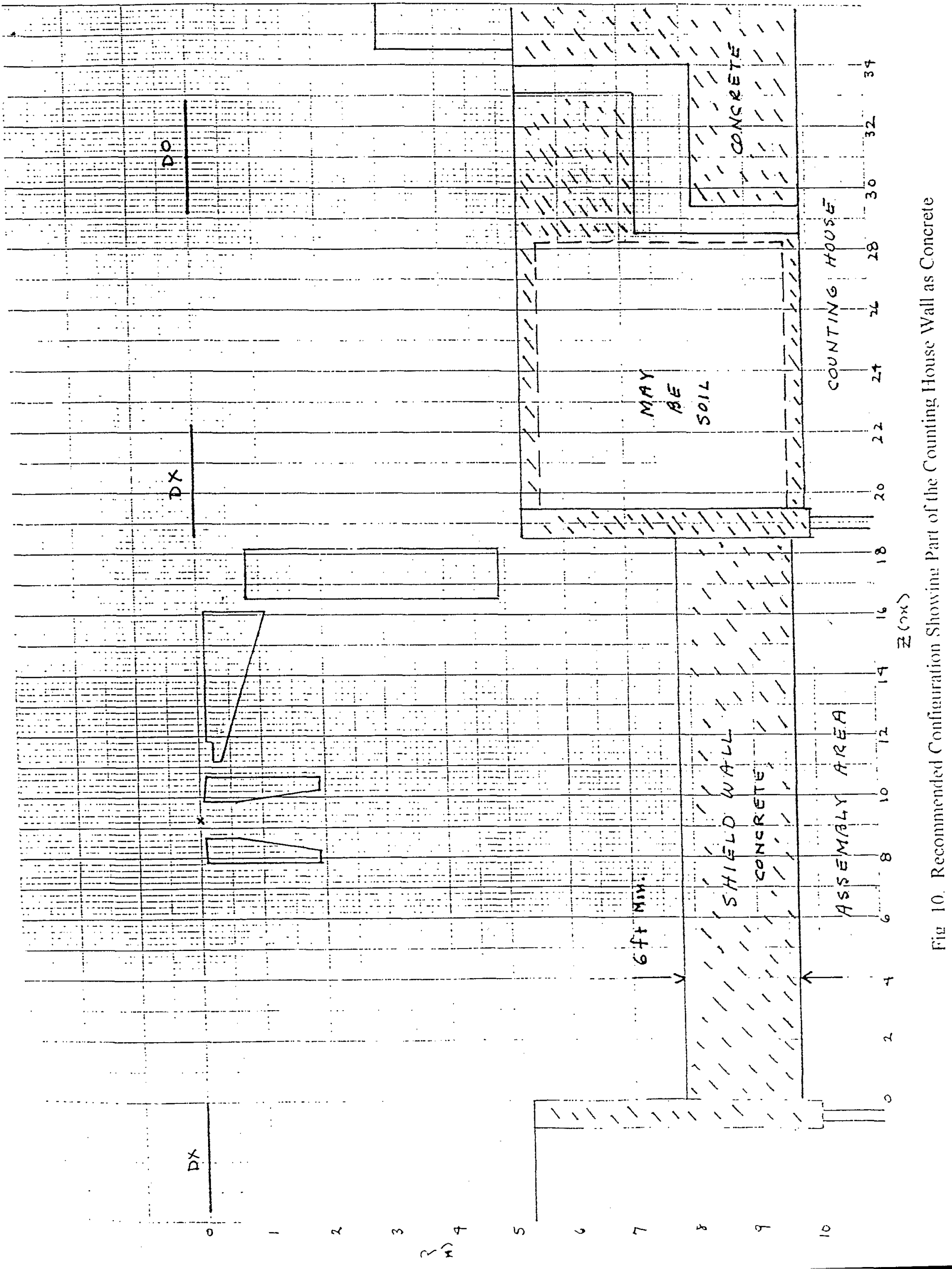


Dr. A.J. Stevens

$1005 \mathrm{~S}$ 
BROOKHAVEN NATIONAL LABORATORY

August, 1995

RHIC DETECTOR NOTE

RHIC/DET Note 13E

Erratum to RHIC/DET Note 13

Estimated Shielding Requirements for the PHENIX Detector

\author{
Alan J. Stevens \\ Brookhaven National Laboratory \\ Upton, NY 11973
}


This Note is an Erratum to RHIC/DET Note 13 entitled "Estimated Shielding Requirements for the PHENIX Detector. ${ }^{1}$ In recent calculations related to the PHENIX shield wall, an error in one of the user-defined CASIM subroutines used in the calculations reported in Ref [1] was discovered. The nominal effect of the error was an underestimate of the star density whose magnitude depended on transverse radius. For the star density at the back of the shield wall assumed $\left(R_{t}=9.4 \mathrm{~m}\right)$, the error would cause an underestimate of the star density by a factor of 1.25 .

Since the calculations reported in Ref [1] were performed, CASIM was moved from a VAX machine (bnlhep) to the Detector Group's IBM 6000 RISC machines which are much faster. Since the nominal statistical error on the star densities in Ref [1] were 15\%, comparable to the error caused by the bug, the shield wall calculations were re-run with bug corrected and with $\sim 4$ times the number of primary $250 \mathrm{GeV} / \mathrm{c}$ protons simulating the faults.

Fig. 1 is a corrected version of Fig. 3 in Ref [1]. For a detailed explanation of this figure the reader is referred to Ref [1]. Briefly, the points in this figure give the azimuthally averaged star density at the back of a $1.6 \mathrm{~m}$ thick concrete wall which begins at a transverse radius of $7.8 \mathrm{~m}$ from the beam line. The circles correspond to a fault on DX where the muon detector shielding is not relevant, i.e., a geometry where only one muon detector exists. The plus signs correspond to a fault on the DX on the opposite side of the 8 o'clock hall where the muon detector adds to the shielding, and the triangles correspond to a fault on the beam pipe at the position of the muon piston. The geometry is shown in Fig. 1 of Ref [1].

The design basis fault is one half of 4 times the design intensity $250 \mathrm{GeV} / \mathrm{c}$ protons interacting on either DX or the piston. If only one muon detector is present, the circles in the revised figure determine the minimum thickness of the shield. The dose equivalent for the design basis fault at $1.6 \mathrm{~m}$ shield wall thickness is:

$$
\begin{aligned}
\text { Maxmrem } / \text { fault } & =2.85 \times 10^{-9} \text { star } / c c / p \times 1.8 \times 10^{-2} \mathrm{mrem} / \mathrm{star} / \mathrm{cc} \times 1.3 \times 1.14 \times 10^{13} \mathrm{p} \\
& =760 \mathrm{mrem}
\end{aligned}
$$

where the 1.3 factor is for an enhancement on the mid-plane caused by the magnetic field of DX and the star density to rem conversion follows the normal practice at RHIC of doubling the neutron quality factor for design purposes. For a $6 \mathrm{ft}$. thick $(1.83 \mathrm{~m})$ shield, this scales to 455 mrem. Since this is below the 500 mrem criteria adopted by the RHIC project the conclusion of Ref [1] remains true, namely that if only one muon detector exists a six $\mathrm{ft}$. thick shield wall of light concrete (or the equivalent) is required.

If two muon detectors are present, the fault on the opposite side DX dominates. In this case the dose equivalent for the design basis fault at $1.6 \mathrm{~m}$ is reduced from the result given above by $1.45 / 2.85$. The $387 \mathrm{mrem}$ at $1.6 \mathrm{~m}$ scales to $454 \mathrm{mrem}$ at a $5 \mathrm{ft}$. wall thickness. Thus here also, the conclusion of Ref [1] remains true; if two muon detectors exist a five ft. thick shield wall of light concrete (or the equivalent) is required. 
Ref [1] also contains calculations pertaining to the counting house region. Here the relevant radius would imply a slightly smaller correction, 1.20 instead of 1.25 . Since the recommendation of Ref [1] was a configuration that contained a factor of 2 safety, these calculations have not been repeated.

In summary, the error discovered in the calculations reported in RHIC/DET Note 13 was small enough that all of the conclusions and recommendations made therein remain unchanged.

\section{$\underline{\text { References/Footnotes }}$}

1. A.J. Stevens, "Estimated Shielding Requirements for the PHENIX Detector," RHIC/DET Note 13, December, 1994.

2. The star density follows $\mathrm{e}^{-\mathrm{t} / \lambda} / \mathrm{R}_{\mathrm{t}}^{2}$. For light concrete $\lambda$ is $.502 \mathrm{~m}$. $\mathrm{R}_{\mathrm{t}}$ is measured to the back of the shield wall whose front location is assumed fixed. 


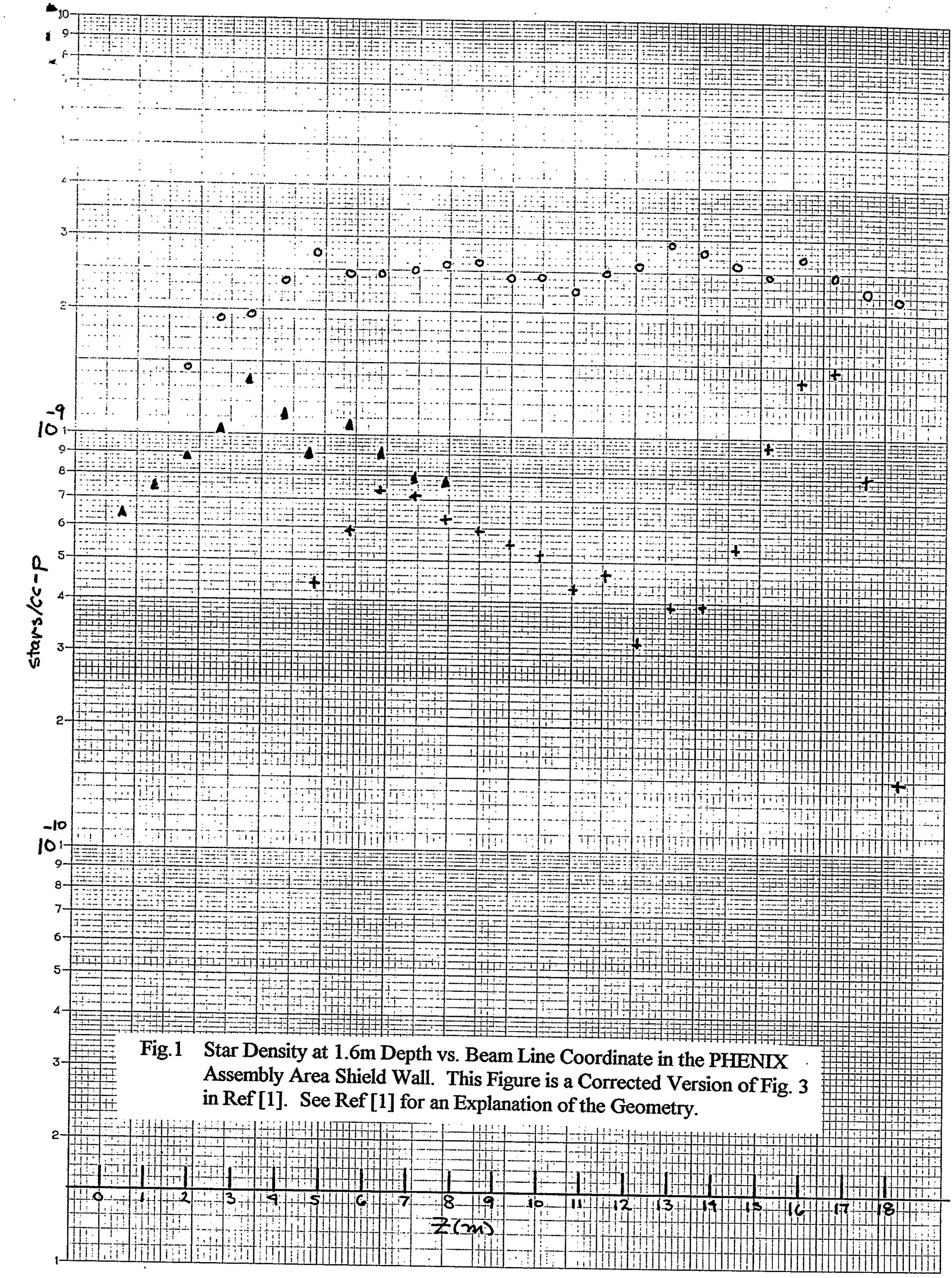

